\title{
The breed prevalence of Dog Erythrocyte Antigen 1.1 in the Onderstepoort area of South Africa and its significance in selection of canine blood donors
}

\author{
L L van der Merwe ${ }^{a}$, L S Jacobson ${ }^{a}$ and G J Pretorius ${ }^{a}$
}

\begin{abstract}
The blood group antigen Dog Erythrocyte Antigen (DEA) 1.1 is clinically the most important canine blood group as DEA 1.1 antibodies are capable of causing acute haemolytic, potentially life-threatening transfusion reactions. Dogs do not have naturally occurring antibodies to DEA 1.1 but are rapidly sensitised by the first incompatible transfusion. The prevalence of DEA 1.1 in the general dog population is estimated at $42-46 \%$. Canine blood donors registered with the Onderstepoort Animal Blood Bank $(n=93)$ as well as potential donors $(n=140)$ were typed for DEA 1.1 using a monoclonal antibody card kit. All dogs came from the Onderstepoort area, near Pretoria, Gauteng province, South Africa. Overall prevalence of DEA 1.1 was $47 \%$. Prevalence was $47 \%$ in purebred dogs and $48 \%$ in mongrels. Distinct breed differences were noted with less than $20 \%$ of German shepherd dogs and Boxers and greater than $75 \%$ of Rottweilers, Great Danes, St Bernards and Dalmations testing DEA 1.1 positive. Knowledge of local breed differences will increase effectiveness of blood donor recruitment.
\end{abstract}

Key words: blood donors, blood transfusion, blood typing, breed prevalence, canine, DEA 1.1, dog, dog erythrocyte antigen, haemolysis.

Van der Merwe L L, Jacobson L S, Pretorius G J The breed prevalence of Dog Erythrocyte Antigen 1.1 in the Onderstepoort area of South Africa and its significance in selection of canine blood donors. Journal of the South African Veterinary Association (2002) 73(2): 53-56 (En.). Department of Companion Animal Clinical Studies, Faculty of Veterinary Science, University of Pretoria, Private Bag X04, Onderstepoort, 0110 South Africa.

\section{INTRODUCTION}

Canine blood group antigens are referred to as dog erythrocyte antigens (DEA). Currently 6 DEA can be routinely identified in blood typing schemes, namely DEA $1.1,1.2,3,4,5$, and $7^{10}$. The antisera for 6 and 8 have been lost. All DEA except DEA 7 are constitutional proteins of the erythrocyte membrane ${ }^{3}$. Canine blood group antigens are inherited according to an independent autosomal dominant model $^{4}$. Only the DEA 1 group occurs as a multiple allele with DEA 1.1 dominant over DEA 1.2 (i.e. DEA 1.1 and 1.2 cannot both be phenotypically present $)^{4}$.

The importance of determining canine blood groups is: (1) determining the prevalence of the antigen in the dog population, (2) determining the prevalence of naturally occurring antibody, (3) determining the effect of the antibody versus the antigen in vivo, and (4) evaluating the immunogenicity of the antigen ${ }^{10,18}$.

Blood transfusion is a clinical therapy not without risk to the patient. The aim of blood transfusion is to provide a maxi-

aDepartment of Companion Animal Clinical Studies, Faculty of Veterinary Science, University of Pretoria, Private Bag X04, Onderstepoort, 0110 South Africa.

E-mail: Ivdmerwe@op.up.ac.za

Received: April 2001. Accepted: March 2002. mum number of viable erythrocytes and to minimise the number of erythrocytes lost due to storage injury or immunemediated destruction. It is also the responsibility of the veterinarian to minimise risks associated with the transfusion of blood. This responsibility includes selection of an appropriate donor animal, aseptic and atraumatic collection procedures, proper storage and correct administration of the blood ${ }^{11}$.

Transfusion reactions may be classified as immunological or non-immunologi $\mathrm{cal}^{11}$. Although the various DEA groups are all capable of causing immune mediated reactions (type II hypersensitivity), the type (haemolytic vs erythrophagocytic) and severity vary greatly ${ }^{10-12}$. Canine blood group antigens also display marked variability in antigenicity and dogs are not equally capable of producing antibodies to these foreign proteins ${ }^{18,20}$. In humans it is essential to know the blood group before a first blood transfusion is given, because life-threatening transfusion reactions can result from pre-formed antibodies against the donor's blood group $^{6}$. This is not the case in dogs. In this species, pre-formed antibodies can occur against DEA 3, 5 and 7, but the titres are generally very weak and the prevalence of pre-formed antibodies low ${ }^{10}$. Weak auto-antibodies cause a delayed removal, over 3-5 days, of DEA 3, 5 and 7 incompatible blood from the circulation ${ }^{18}$. DEA 1.1 and DEA 4 are the most antigenic of the DEA, with dogs usually developing substantial titres within 4-40 days in negative recipients $^{6,17,18}$. The antibody against DEA 4 , a blood group antigen present in $98 \%$ of the population, is benign, causing no erythrocyte destruction, whereas that developed against DEA 1.1, a blood group antigen present in $42-46 \%$ of the general dog population, is a haemolysin ${ }^{6,17,18}$. DEA 1.1-, 1.2- and 7-negative donors are considered universal donors by some authors. For practical purposes, however, DEA 1.1 status is the most important issue in selecting canine blood donors ${ }^{8,9,10}$.

As no pre-formed antibodies exist against DEA 1.1 or 1.2, a first transfusion with DEA 1-positive blood in a negative recipient will not result in a transfusion reaction ${ }^{18}$. These animals will, however, be sensitised to subsequent transfusions of DEA 1-positive blood. Alloantibodies do, however, develop 1-2 weeks after even the first DEA 1.1 incompatible transfusion, resulting in the accelerated removal of transfused cells ${ }^{18}$. DEA 1.1 is one of the most reactive, and clinically the most important, of all the DEA groups. Incompatibility causes an acute ( $<12 \mathrm{hrs})$ haemolytic transfusion reaction ${ }^{6,9,17,18}$. DEA 1.1 antibodies are capable of causing agglutination as well as complement activation and opsonisation of targeted erythrocytes ${ }^{18}$. Complement activation results in acute intravascular haemolysis and opsonisation facilitates erythrophagocytosis. The effect of DEA 1.1 transfusion to a sensitised individual has been experimentally followed ${ }^{9,16,17}$. When small volumes of erythrocytes were transfused, all were removed from the circulation within $5 \mathrm{~min}$ of administration while larger volumes $(100-150 \mathrm{ml})$ took up to $24 \mathrm{~h}$. Maximum free haemoglobin was measured within 20 min of administration. Damaged erythrocyte membranes expose the immune system to phospholipids, which are thromboplastic and cause activation of the systemic inflam- 
matory response system. In a healthy dog, the acute haemolytic transfusion reaction, while appearing clinically severe, was seldom fatal. This has not, however, been determined in animals that are already compromised as in babesiosis, sepsis, surgery or haemorrhagic shock.

DEA 1.2 is less reactive, generally inducing agglutination and delayed destruction of targeted erythrocytes ${ }^{6,17}$. Less commonly DEA 1.2 is capable of causing haemolysis. The reaction is usually subacute $(12-24 \mathrm{~h})$ with clinical haemolysis usually undetectable ${ }^{6}$.

Based on the reported prevalence of DEA $1.1(42-46 \%)$, approximately $23 \%$ of random transfusions have the potential to sensitise recipients ${ }^{20}$. The risk of a transfusion reaction in a recipient receiving a second random transfusion is $15 \%^{6,20}$. This risk is especially relevant in South Africa where patients are more likely to receive 2 or more blood transfusions in their lifetime owing to the prevalence of babesiosis.

The reported prevalence of each of the canine blood group factors appears relatively consistent between widely separated random dog populations ${ }^{2,6,10,12,15,18}$. This, as well as the consistency between mongrels and purebred dogs, has led authors to speculate that blood group genes and antigens are an 'old evolutionary heritage $e^{4,17}$. Most authors report a general population prevalence, and few have mentioned breed prevalence of DEA 1.1. There is, however, some evidence of breed differences. Beagles were reported to have $43.4 \%$ prevalence of DEA 1.1 and Labrador retrievers $29 \%^{2}$. A study performed in Brisbane to study the canine A blood group system (DEA 1) evaluated $968 \mathrm{dogs}$ of various breeds. Prevalence of DEA 1.1 in the total and the mongrel populations $(n=410)$ was $42 \%$, but breed differences were apparent. The prevalence of DEA 1.1 was: Afghan $84 \%$, St Bernard $63 \%$, Labrador $59 \%$, Greyhound $46 \%$, German shepherd dog $30 \%$, Boxer $25 \%$, Australian cattle dog $23 \%$ and Border collie $16 \%{ }^{19}$.

The Onderstepoort Veterinary Academic Hospital administers a large number of canine blood transfusions, most of which are provided to dogs with babesiosis and, to a much lesser extent, elective surgery and trauma. The Onderstepoort Animal Blood Bank provides blood for 60 transfusions per month in summer; in 2000 it provided 450 transfusions in a total hospital population of $6399(7 \%)$. Transfusions are also regularly administered by private practitioners, usually for life threatening babesiosis ${ }^{5}$. Historically, canine blood donors in South Africa have not been typed, at least in part due to lack of perceived need but also due to technical constraints.

Results of transfusions are generally considered very good ${ }^{14}$. However, observations have not been consistent enough to establish whether clinically significant transfusion reactions are a regular event. Three major potential problem areas exist: (1) deaths from acute haemolytic transfusion reactions may be misinterpreted as death due to the underlying disease process; (2) delayed transfusion reactions resulting in disappointingly low haematocrits following transfusion may be attributed to the vagaries of the disease process; and (3) transfusion reactions in dogs with concurrent, usually secondary, immune-mediated haemolytic anaemia, where, again, disappointing results might be attributed to the disease rather than the transfusion. Little real progress can be made without basic information about the blood types being transfused. Until recently, the only method for detecting canine blood group antigens has been the use of polyclonal antibody serology, which is limited to specialised laboratories. Complete canine serological blood typing is not available in South Africa and reagents for these tests are not commercially available. It is generally accepted, however, that typing for all blood groups is not necessary and that DEA 1.1 status is most important. A monoclonal card test was developed to type dogs for DEA 1.1, and is commercially available ${ }^{1}$.

The use of a DEA 1.1-negative donor for a first transfusion and additional crossmatching (major) with a second transfusion is considered practical and safe $e^{8,9,18,20}$. Most dogs requiring transfusion in South Africa are severely anaemic babesiosis cases that urgently require red blood cells. An added complication is the relatively common occurrence of secondary immune-mediated haemolytic anaemia (IMHA), diagnosed by positive in-saline agglutination, and Coombes positivity associated with babesiosis ${ }^{7,13}$. This factor, together with the haemolytic nature of the primary disease, reduces the usefulness and practicality of crossmatching in babesiosis.

The aim of this study was to determine the DEA 1.1 status in current and potential donors of the Onderstepoort Animal Blood Bank, thus determining its prevalence and breed distribution.

\section{MATERIALS AND METHODS}

Samples for DEA 1.1 determination were collected from 234 existing and potential blood donors drawn from the service area of the Onderstepoort Veterinary Academic Hospital, just north of Pretoria, Gauteng province. The existing donor group $(n=93)$ consisted of 85 outpatient donors and 8 Greyhound inpatient donors. The potential donor group consisted of hospital out- and inpatients $(n=141)$. Inclusion criteria for potential donors were: large-breed dogs with an adult mass of over $30 \mathrm{~kg}$; no previous blood transfusions; no breeding bitches; clinically healthy; haematologically normal and free of any blood-borne parasites detectable on thin bloodsmear evaluation.

Blood was collected into a 3-ml EDTA vacutainer tube from the cephalic or jugular vein. The sample was stored at $4{ }^{\circ} \mathrm{C}$ and tested within $24 \mathrm{~h}$. DEA 1.1 status was determined using the RapidVet ${ }^{\mathrm{TM}}-\mathrm{H}$ (Canine 1.1) test (DMS Laboratories, Flemington, New Jersey, USA). All samples were first evaluated for autoagglutination (in-saline agglutination test) and those showing positive agglutination were not tested further.

The RapidVet ${ }^{\mathrm{TM}}-\mathrm{H}$ assay is based on an agglutination reaction that occurs when erythrocytes containing DEA 1.1 on the surface membrane react with a murine monoclonal antibody against DEA 1.1 which is lyophilised onto the test card. DEA 1.1-positive samples cause a coarse agglutination $(2+)$ reaction, while DEA 1.1-negative samples cause no reaction. Each card contains a positive control, negative control and patient sample well. The test procedure was performed according to the manufacturers' instructions using controls supplied. Cards were allowed to dry and stored as a permanent record.

To evaluate the frequency of breeds tested and the frequency within the hospital population hospital records over a 12-month period were searched for dogs with a mass $\geq 30 \mathrm{~kg}$, and the breed frequencies calculated.

\section{RESULTS}

One of the 234 samples showed agglutination and was excluded. Of the 233 dogs typed, 23 were mongrels and 210 were purebred. Forty-seven percent of the dogs (110/233) tested DEA 1.1-positive. Of the purebred dogs, $47 \%$ (99/210) tested DEA 1.1-positive and of the 23 mongrels, $48 \%(11 / 23)$ tested positive.

Despite this uniform overall picture, there were striking breed differences (Fig. 1). Breeds evaluated in reasonable numbers ( $>8 /$ breed) were the German shepherd dog (55), Boerboel (33), Rottweiler (23), Labrador retriever (20), Bull mastiff (9), Boxer (8), and Greyhound (8). Approximately three-quarters of Boerboels $(73 \%)$ and Rottweilers $(78 \%)$ were positive. All Greyhounds were negative, as were most Boxers (88 \%), German 


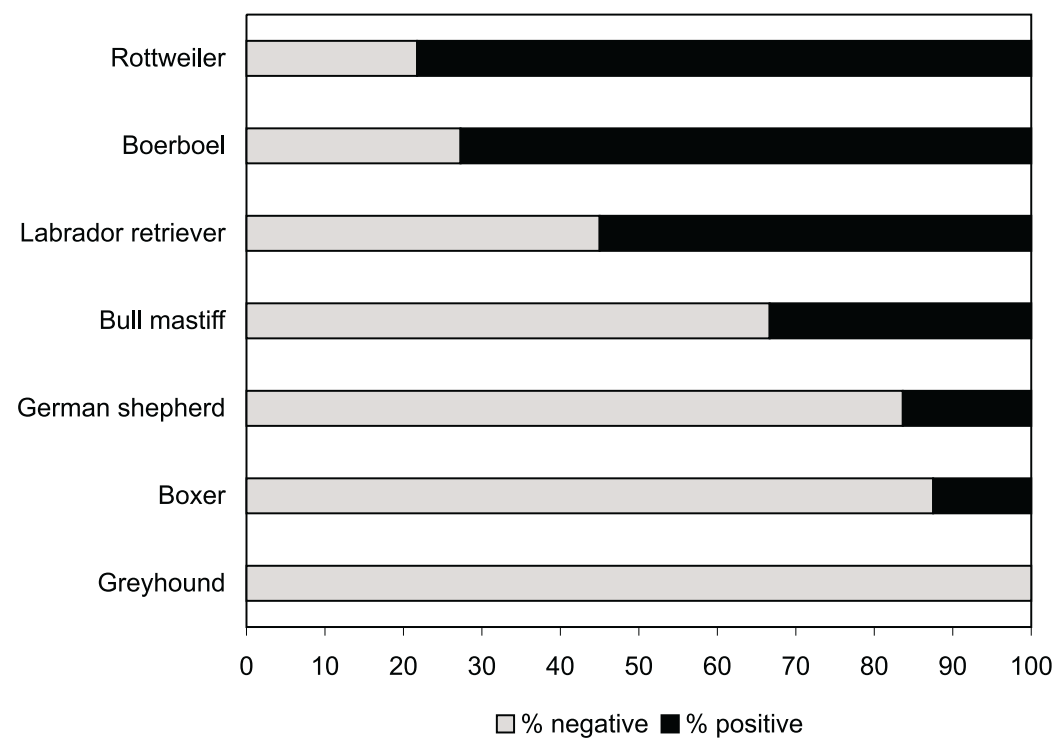

Fig 1: Proportion of dogs negative and positive for DEA 1.1 in breeds where at least 8 dogs were tested.

shepherd dogs (84\%), and Bull mastiffs $(67 \%)$. Of the larger groups, only Labrador retrievers fitted the more general picture, with similar numbers of positive and negative reactors. Of the smaller groups where at least 2 dogs were tested, both Weimaraners were negative and all Bouviers (3), Dalmations (4), Great danes (5) and St Bernards (2) were positive. Owing to the small numbers in individual groups, statistical analysis was not performed.

The breed frequency distributions indi- cated that the population tested was generally representative of the hospital population (Table 1).

\section{DISCUSSION}

The frequency of DEA 1.1 in this population of dogs in the northern part of South Africa was similar to frequencies reported in dog populations elsewhere ${ }^{2,10,15,18}$. However, within individual breeds, the picture changed markedly, with very low DEA 1.1 frequencies in some breeds and very high frequencies in others. No effort was made to determine the lineage of the dogs, none were from breeding kennels/programmes, and any shared parentage would have been coincidental. The DEA 1.1 prevalence in individual breeds in our study differed from the study performed in Brisbane. Small isolated populations of animals would, however, be expected to show some variations and care must be taken with the interpretation and extrapolation of data from these small groups ${ }^{4}$.

These results strongly support the utility of seeking blood donors from specific breeds to obtain our initial goal of a DEA 1.1-negative donor population. Ideally, donor breeds should be large, easy to train, common in the local dog population with a low prevalence of DEA 1.1. The German shepherd dog stands out as a breed that fits all these criteria. Other breeds that had low prevalence of DEA 1.1 are not common in the Onderstepoort area, although enough Boxers are seen to make this a potentially useful donor breed. Owners or breed societies of less popular breeds could be individually approached to have their dogs considered as donors. Popular breeds that appear to be undesirable as blood donors in this area are Boerboels (a mastiff-type breed), and Rottweilers. An additional use of these data is to inform pet owners of the likelihood of particular breeds being positive or negative, which would

Table 1: Breed prevalence of DEA 1.1 in dogs in the Onderstepoort area, Gauteng province, South Africa.

\begin{tabular}{|c|c|c|c|c|c|}
\hline Breed & Number & DEA 1.1-positive & $\begin{array}{l}\text { Percentage } \\
\text { positive }\end{array}$ & $\begin{array}{l}\text { Frequency in } \\
\text { sample (\%) }\end{array}$ & $\begin{array}{c}\text { Frequency (\%) in } \\
\text { hospital population* }\end{array}$ \\
\hline Belgian shepherd & 1 & 0 & 0 & 0.4 & 0.1 \\
\hline Greyhound & 8 & 0 & 0 & 3.4 & 0.0 \\
\hline Weimaraner & 2 & 0 & 0 & 0.9 & 0.8 \\
\hline Boxer & 8 & 1 & 13 & 3.4 & 2.5 \\
\hline German shepherd & 55 & 9 & 16 & 23.6 & 20.7 \\
\hline Bull terrier & 4 & 1 & 25 & 1.7 & 1.5 \\
\hline Staffordshire terrier & 4 & 1 & 25 & 1.7 & 0.3 \\
\hline Bull mastiff & 9 & 3 & 33 & 3.9 & 4.0 \\
\hline Border collie & 5 & 2 & 40 & 2.1 & 0.2 \\
\hline Pitbull terrier & 5 & 2 & 40 & 2.1 & 1.0 \\
\hline Rhodesian ridgeback & 5 & 2 & 40 & 2.1 & 3.4 \\
\hline Mongrel & 23 & 11 & 48 & 9.9 & 14.5 \\
\hline Pointer & 2 & 1 & 50 & 0.9 & 0.2 \\
\hline Labrador retriever & 20 & 11 & 55 & 8.6 & 6.0 \\
\hline Doberman & 5 & 3 & 60 & 2.1 & 2.9 \\
\hline Boerboel & 33 & 24 & 73 & 14.2 & 18.5 \\
\hline Husky & 4 & 3 & 75 & 1.7 & 0.0 \\
\hline Rottweiler & 23 & 18 & 78 & 9.9 & 14.7 \\
\hline Anatolian shepherd & 1 & 1 & 100 & 0.4 & 0.0 \\
\hline Bernese mountain dog & 1 & 1 & 100 & 0.4 & 0.1 \\
\hline Bouvier & 3 & 3 & 100 & 1.3 & 0.6 \\
\hline Dalmation & 4 & 4 & 100 & 1.7 & 0.4 \\
\hline Golden retriever & 1 & 1 & 100 & 0.4 & 0.7 \\
\hline Great Dane & 5 & 5 & 100 & 2.1 & 2.8 \\
\hline St Bernard & 2 & 2 & 100 & 0.9 & 1.1 \\
\hline
\end{tabular}

DEA $=$ dog erythrocyte antigen.

${ }^{*}$ Population data taken from hospital database of all patients $>30 \mathrm{~kg}$, including both inpatients and outpatients, in 2000. 
affect their status as potential recipients of blood.

The need for transfusing only DEA 1.1negative blood should be apparent. This would ensure the provision of a highquality service to the client and maximise the life-span of transfused erythrocytes in the patient while at the same time reducing the risk of an iatrogenic haemolytic crisis. The test is rapid, simple and relatively inexpensive and is currently available through the Onderstepoort Animal Blood Bank. If DEA 1.1 typing is not possible, selection of donors from breeds with a low frequency of DEA 1.1 would be prudent, bearing in mind that regional variations in frequencies might exist. Most practices could, however, establish and manage an outpatient blood donor scheme with all donors typed DEA 1.1negative.

In conclusion, the DEA 1.1 frequency in a group of 233 dogs from the Onderstepoort area of South Africa was consistent with previous reports elsewhere in the world, marked breed differences were however found. Knowledge of breed differences is very useful for efficient recruitment of typed blood donors, considering that DEA 1.1 could be the only antigen of practical clinical importance in canine blood transfusion.

\section{ACKNOWLEDGEMENTS}

This project was funded by the Faculty Research Fund and the Onderstepoort Veterinary Academic Hospital, Faculty of Veterinary Science, University of Pretoria. We gratefully acknowledge their support. The project was approved by the Animal Use and Care and Research Committees of the Faculty of Veterinary Science, Uni- versity of Pretoria (registered project 36.5.365). LSJ was funded during the period of the study by the Technology and Human Resources for Industry Programme (THRIP), a partnership programme funded by the Department of Trade and Industry (DTI) and managed by the National Research Foundation. We thank Mrs Carien Muller for her assistance with the blood typing.

\section{REFERENCES}

1. Andrews G A, Chavey P S, Smith J E 1992 Production, characterization, and applications of a murine monoclonal antibody to dog erythrocyte antigen 1.1. Journal of the American Veterinary Medical Association 201: 1549-1552

2. Bell K 1983 The blood groups of domestic animals. In Agar N S, Board, P G (eds) Red blood cells of domestic mammals. Elsevier Science Publishers, Amsterdam: 133-164

3. Bull R W 1982 Antigens, graft rejections, and transfusions. Journal of the American Veterinary Medical Association 181: 1115-1119

4. Cohen C, Fuller J L 1953 The inheritance of blood types in the dog. Journal of Heredity 44: 225-228

5. Collet M G 2000 Survey of canine babesiosis in South Africa. Journal of the South African Veterinary Association 71: 180-186

6. Dudok de Wit C, Coenegracht N A C J, Poll P H A, Van der Linde J D 1967 The practical importance of blood groups in dogs. Journal of Small Animal Practice 8: 285-289

7. Farwell G E, Legrand E K, Cobb C 1982 Clinical observations on Babesia gibsoni and Babesia canis infections in dogs. Journal of the American Veterinary Medical Association 180: 507-511

8. Giger U 2000 Blood typing and crossmatching to ensure compatible transfusions. In Bonagura, J D (ed.) Kirks current veterinary therapy XIII, small animal practice. W B Saunders, Philadelphia: 396-400

9. Giger U, Gelens, C J, Callan M B, Oakley D A 1995 An acute hemolytic transfusion reaction caused by dog erythrocytic anti- gen 1.1 incompatibility in a previously sensitized dog. Journal of the American Veterinary Medical Association 206: 1358-1362

10. Hale A S 1995 Canine blood groups and their importance in Veterinary Medicine. Veterinary Clinics of North America: Small Animal Practice 25: 1323-1332

11. Harrell K A, Kristensen A 1995 Canine transfusion reactions and their management. Veterinary Clinics of North America: Small Animal Practice 25:1333-1364

12. Harrell K, Parrow, J, Kristensen A 1997 Canine transfusion reactions. Part 1 . causes and consequences. The Compendium of Continuing Education for the Practising Veterinarian 19: 181-190

13. Jacobson L S, Lewis D C 1994 Blood transfusion in dogs, with special reference to babesiosis. Vetmed 7: 81-88

14. Leisewitz A L, Guthrie A J, Berry W L 1996 Evaluation of the effect of whole-blood transfusion on the oxygen status and acidbase balance of Babesia canis infected dogs using the oxygen status algorithm. Journal of the South African Veterinary Association 67: 20-26

15. Stormont C, Suzuki Y 1980 Canine blood groups. Technical Information Center, US Department of Energy, Springfield, Virginia

16. Surgenor D M 1974 Erythrocytes and blood coagulation. Thrombosis Diathesis et Haemorrhagica 32: 247-259

17. Swisher S N, Young L E 1961 The blood grouping systems of dogs. Physiological Review 41: 495-520

18. Swisher S N, Young L E, Trabold N 1962 In vitro and in vivo studies of the behaviour of canine erythrocyte-isoantibody systems. Annals of the New York Academy of Sciences 97: 15-25

19. Symons M, Bell K 1991 Expansion of the canine A blood group system. Animal Genetics 22: 227-235

20. Young L E, O'Brien W A, Swisher S N, Miller G, Yuile C L 1952 Blood groups in dogs - their significance to the veterinarian. American Journal of Veterinary Research 13: 207-213 